\title{
Do Collaborative Care Managers and Technology Enhance Primary Care Satisfaction with Care from Embedded Mental Health Providers?
}

\author{
Lucinda B. Leung, MD, PhD, MPH ${ }^{1,2}$, Alexander S. Young, MD, MSHS ${ }^{1,3,4,5}$, \\ Leonie Heyworth, MD, MPH' ${ }^{6,7}$, Danielle Rose, $P h D^{7}$, susan Stockdale, $P h D^{7}$, \\ A. Laurie Graaff, RN, MBA, MS-ISM ${ }^{8}$, Timothy R. Dresselhaus, MD, MPH ${ }^{7,9}$, and \\ Lisa $V$. Rubenstein, MD, MSPH 2,5,10
}

\begin{abstract}
'Center for the Study of Healthcare Innovation, Implementation, \& Policy, VA Greater Los Angeles Healthcare System, Los Angeles, CA, USA; ${ }^{2}$ Division of General Internal Medicine and Health Services Research, UCLA David Geffen School of Medicine, Los Angeles, CA, USA; ${ }^{3}$ VA VISN 22 Mental IIIness Research, Education, and Clinical Center, Los Angeles, CA, USA; ${ }^{4}$ Department of Psychiatry and Biobehavioral Sciences, UCLA Semel Institute for Neuroscience and Human Behavior, Los Angeles, CA, USA; ${ }^{5}$ RAND Corporation, Santa Monica, CA, USA; ${ }^{6}$ Office of Connected Care/Telehealth, Department of Veterans Affairs Central Office, Washington, DC, USA; ' Department of Medicine, UCSD School of Medicine, San

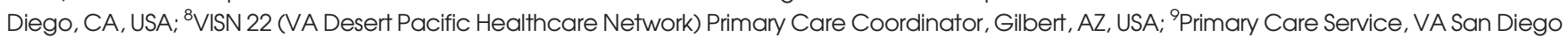
Healthcare System, San Diego, CA, USA; ${ }^{10}$ Department of Health Policy \& Management, UCLA Fielding School of Public Health, Los Angeles, CA, USA.
\end{abstract}

BACKGROUND: To improve mental health care access, the Veterans Health Administration (VA) implemented Primary Care-Mental Health Integration (PC-MHI) in clinics nationally. Primary care clinical leader satisfaction can inform model implementation and may be facilitated by collaborative care managers and technology supporting cross-specialty collaboration.

OBJECTIVE: (1) To determine primary care clinical leaders' overall satisfaction with care from embedded mental health providers for a range of conditions and (2) to examine the association between overall satisfaction and two program features (care managers, technology).

DESIGN: Cross-sectional organizational survey in one VA region (Southern California, Arizona, and New Mexico), 2018.

PARTICIPANTS: Sixty-nine physicians or other designated clinical leaders in each VA primary care clinic $(94 \%$ response rate).

MAIN MEASURES: We assessed primary care clinical leader satisfaction with embedded mental health care on four groups of conditions: target, non-target mental health, behavioral health, suicide risk management. They additionally responded about the availability of mental health care managers and the sufficiency of information technology (telemental health, e-consult, instant messaging). We examined relationships between satisfaction and

Prior Presentations This work was awarded $1^{\text {st }}$ Place Scientific Abstracts Poster Presentation at the Society of General Internal Medicine (SGIM) 2019 California-Hawaii Regional Meeting, Los Angeles, CA, and selected for a Poster Walk and Talk at the SGIM Annual Meeting (May 9, 2019), Washington, DC.

Electronic supplementary material The online version of this article (https://doi.org/10.1007/s11606-020-05660-1) contains supplementary material, which is available to authorized users.

Received March 4, 2019

Revised August 6, 2019

Accepted December 27, 2019

Published online June 17, 2020 the two program features using $\chi^{2}$ tests and multivariable regressions.

KEY RESULTS: Most primary care clinical leaders were "very satisfied" with care for targeted anxiety $(71 \%)$ and depression (69\%), but not for other common conditions (37\% alcohol misuse, 19\% pain). Care manager availability was significantly associated with "very satisfied" responses for depression $(p=.02)$ and anxiety care by embedded mental health providers $(p=.02)$. Highly rated sufficiency of communication technology (only 19\%) was associated with "very satisfied" responses to suicide risk management ( $p=.002)$.

CONCLUSIONS: Care from embedded mental health providers for depression and anxiety was highly satisfactory, which may guide improvement among less satisfactory conditions (alcohol misuse, pain). Observed associations between overall satisfaction and collaborative care features may inform clinics on how to optimize staffing and technology based on priority conditions.

KEY WORDS: primary care; mental health; care management; collaborative care; health information technology; health informatics; Veterans.

$\mathrm{J}$ Gen Intern Med 35(12):3458-64

DOI: $10.1007 / \mathrm{s} 11606-020-05660-1$

(c) Society of General Internal Medicine (This is a U.S. government work and not under copyright protection in the U.S.; foreign copyright protection may apply) 2020

\section{INTRODUCTION}

Striving to offer Veterans "integrated, comprehensive, and coordinated" care, ${ }^{1}$ the Veterans Health Administration (VA) underwent two major national initiatives to transform primary care into patient-centered team-based care models: (1) Primary Care-Mental Health Integration (PC-MHI) ${ }^{2}$ beginning in 2007 and (2) VA's Patient-Centered Medical Home (termed 
Patient-Aligned Care Teams or PACT) in 2010. ${ }^{3}$ PC-MHI represents the largest US implementation of integrated mental health services (nearly 400 clinics nationally), largely based on collaborative care models. ${ }^{4}$ These programs embedded mental health specialists (e.g., psychologists, social workers) in primary care to deliver short-term evidence-based mental health treatments. ${ }^{5}$ It also included nurse care managers who facilitated care coordination and cross-specialty collaboration, per Translating Initiatives in Depression into Effective Solutions (TIDES) and Behavioral Health Laboratory (BHL) ${ }^{6,7}$ implementation studies. PC-MHI, along with PACT, enhanced primary care staffing and aimed to care for most patients with low-to-moderate complexity mental health conditions within primary care. ${ }^{8}$ In the decade of VA practice transformation to integrate primary and mental health care, program implementation has focused on mental health specialist feedback with less input from primary care leaders and providers, whose engagement is critical. ${ }^{9}$

Although distinct from technical quality of care ${ }^{10}$, primary care clinical leader satisfaction with PC-MHI services may inform model implementation and improvement. All large primary care sites, serving the large majority of Veterans, are required to have co-located mental health specialists and/or care managers collaboratively supporting primary care providers, but ${ }^{11,12}$ PC-MHI clinical staff composition and roles can vary. This is particularly true for small sites where implementation is voluntary, which make up the majority of clinics but serve a minority (fewer than 5000) of patients annually. ${ }^{13}$ Assessing how well primary care providers think their local PC-MHI programs are meeting patient needs is critical for enhancing program effectiveness and for guiding future research on integrated mental health care models.

Although collaborative care programs have been viewed favorably by patients and providers in randomized controlled trials, ${ }^{14}$ in safety net clinics, ${ }^{15}$ and in the VA, ${ }^{16}$ none of these studies address primary care satisfaction with PC-MHI as implemented in real-world clinical operations. Among the few studies addressing primary care views, a survey study of over 800 primary care physicians in one Canadian region found that less than half were satisfied with the quality of mental health care provided in primary care, and that timely access to mental health specialists was most commonly cited as critical to its improvement. ${ }^{17}$ Interviews with VA clinic leaders and frontline primary care and mental health clinicians conducted within 16 clinics across 8 health care systems in 2012 identified communication barriers between specialties and that time pressures impeded relationship building. ${ }^{18}$ Later analyses showed that personal coordination between both specialties was necessary for resolving barriers related to collaborative care implementation. ${ }^{19}$ Few studies, however, have evaluated which clinical conditions are perceived by primary care leaders and providers as being adequately addressed by PC-MHI, or which conditions required greater specialty collaboration and may be facilitated by program features.
While collaboration between primary care and mental health is crucial to PC-MHI implementation, ${ }^{20}$ tools for collaboration have not been explicitly linked with improved primary care-rated satisfaction across clinical conditions. Two program features aimed at supporting collaboration are traditional care managers for mental health needs ${ }^{6,7}$ and newer information communication technology. ${ }^{21}$ Recognizing that on-site mental health staff availability and warm handoffs may not always be feasible, the VA has invested heavily in technology, including electronic consultation and virtual (not in-person) mental health treatments. ${ }^{22}$ VA's primary care and mental health electronic records have been integrated for several decades, and over this time VA primary care providers and mental health specialists have also had access to real-time instant messaging. ${ }^{23}$ Additionally, starting 2014, the VA awarded workload credit for asynchronous electronic referral and consultation embedded within patient health records. ${ }^{24}$ Recently, telehealth services (i.e., virtual visits) have received legislative and resource support (i.e., 2018 "Anywhere to Anywhere" initiative which allows VA physicians to provide telehealth services to Veterans regardless of state regulations) ${ }^{22}$ to better engage Veterans in mental health care. ${ }^{25}$ While these technologies are not limited to only primary care and mental health use, we know little about how primary care rates the sufficiency of these technologies in PC-MHI programs. ${ }^{26}$

We partnered with primary care leadership in one regional network to design and field a survey of primary care clinical leaders across one multi-state VA administrative region as part of a quality improvement effort initiated by local and regional leadership. The VA primary care clinics represented in our study boast among the highest in penetration of PC-MHI services nationally (i.e., over one in ten primary care patients have received PC-MHI care). Study goals were twofold: (1) to determine primary care clinical leaders' overall satisfaction with care from embedded mental health providers for a range of conditions and (2) to examine the association between overall satisfaction and two program features ${ }^{27}$ - perceived availability of care managers and sufficiency of information technology.

\section{METHODS}

\section{Study Design and Participants}

We conducted a cross-sectional organizational survey on PCMHI services and received responses from key informants for 65 of 69 primary care clinics in Southern California, Arizona, and New Mexico, from February to May 2018 (94\% response rate). While there were 76 clinics that provided primary care services to VA patients in this region during the study period, we excluded seven clinics, including sites that were specialtybased (e.g., HIV, homeless) and Indian Health Services-operated, as previously documented. ${ }^{13}$ Electronic surveys were completed online by each clinic's designated primary care clinical leader. Leaders were frontline full-time VA clinicians (e.g., physicians, nurse practitioners), responsible for overseeing clinical operations, and most knowledgeable about 
medical staffing and technology use within his/her clinic's workflow. We received and analyzed responses from leaders of 7 VA hospital- and 58 community-based primary care clinics, of which only three reported no available PC-MHI services. ${ }^{13}$ On average, these 65 clinics were 73 miles from affiliated VA hospitals and cared for 6437 primary care patients, who were at slightly lower than average clinical risk (Nosos $=0.9[.2]) .^{13}$

\section{Survey Design and Measures}

This study examined responses for three sets of survey questions about PC-MHI programs (Appendix). Questions were irrelevant for three clinics because they reported having neither embedded mental health providers or care managers available for mental health needs. Individual item response rates ranged from 85 to $97 \%$.

Main outcomes were primary care clinical leader-rated satisfaction with various mental health care services provided by embedded specialists (e.g., availability of care, quality of care, communication related to care). Based on the skewed distribution of responses, we dichotomized the 5-point ordinal scale into "very satisfied" versus all other response options. We assessed satisfaction for (1) long-time target PC-MHI conditions (depression, anxiety, alcohol misuse/problem drinking), (2) non-target mental health conditions (posttraumatic stress disorder [PTSD], serious mental illness other than PTSD [schizophrenia, bipolar disorder], substance misuse/ illicit drug problems, mental health symptoms related to military sexual trauma and/or intimate partner violence), (3) other mental health-linked behavioral health issues (sleep problems, complex high medical needs, disruptive behavior, medication non-adherence, non-adherence to necessary clinical care other than medications, pain), and (4) suicide risk management.

The first key independent variable was primary care clinical leader-reported availability of nurse care managers who liaison between primary care and mental health specialists (either on-site or off-site, and through PC-MHI or PACT). We chose to examine the availability of care managers, in order to explore PC-MHI features that facilitate collaboration between primary care provider and existing mental health specialists. In a previous work, we reported that $77 \%$ of study clinics reported available care managers for mental health needs, which may occur on- or off-site and may be funded by primary care and/or mental health resources. ${ }^{13}$

The second key independent variable was primary care clinical leader-reported sufficiency of PC-MHI information communication technology, which may similarly facilitate collaboration between primary care and mental health specialists. In a previous work, we found that the majority of primary care clinical leaders reported that in-person PC-MHI collaboration, such as "warm handoffs" and same-day consultation, were readily assessible. ${ }^{13}$ Here, we considered technological innovations that enhanced collaboration through information sharing, specifically virtual visits and consultation platforms, ${ }^{28}$ and then developed a scale comprised of three sets of survey questions to measure primary care-perceived sufficiency for such technologies (Cronbach $\alpha=$ 0.81 ) (Fig. 1). Primary care clinical leader-rated sufficiency for (1) office space or tools for telemental health (virtual visit) capability, (2) electronic referral/consultation to PC-MHI (e-consult), and (3) real-time electronic communication (instant messaging) with PCMHI staff on a 5-point ordinal scale. Responses from a 5-point ordinal scale were dichotomized as "always sufficient" versus all other responses options and scaled as follows: low (0 technology components reported as "always sufficient"), medium (1-2 components), and high (all 3 components).

Organizational characteristics, such as clinic size (total number of empaneled primary care patients), were obtained from VA administrative data sources. To adjust for case-mix, we obtained Nosos risk scores, which are calculated by the VA to adjust for patient age, gender, physical and mental health diagnoses, pharmacy records, VA priority status (e.g., having a service-connected disability), and VA-computed costs. ${ }^{29} \mathrm{An}$ online web mapping service was used to calculate geographical distance (mileage) from the affiliated VA hospital to each primary care clinic.

\section{Statistical Analysis}

Consistent with the survey's unit of analysis, we conducted analyses at the primary care clinic level. First, we calculated the percentage of primary care clinical leaders reporting they were "very satisfied" with PC-MHI care for target, non-target, and behavioral health conditions or concerns, and reporting "always (and usually) sufficient" for telemental health resources, e-consult, and instant messaging with PC-MHI staff. We then used $\chi^{2}$ statistics to assess associations between (1) primary care clinical leader-rated satisfaction and availability of care manager for mental health needs and (2) primary care clinical leader-rated satisfaction and availability of PC-MHI information communication technology. Finally, we used logistic regressions to examine these relationships, controlling for clinic size, distance to VA hospital, and case-mix. In sensitivity analyses, we examined relationships with outcome measures (1) to each technology component (telemental health, e-consult, instant messaging) and (2) to both key independent variables concurrently in the same model. We reported all individual $p$ values (test statistics and confidence intervals), as no mathematical correction was made for multiple comparisons. For all models, we determined significance using a 2-tailed alpha of 0.05 and analyzed data in Stata 15.0. The VA Greater Los Angeles Institutional Review Board provided a waiver for this non-research, quality improvement effort.

\section{RESULTS}

\section{Primary Care Clinical Leaders' Satisfaction with Care from PC-MHI Providers}

The majority of primary care clinical leaders were "very satisfied" with care for two of three target PC-MHI conditions 


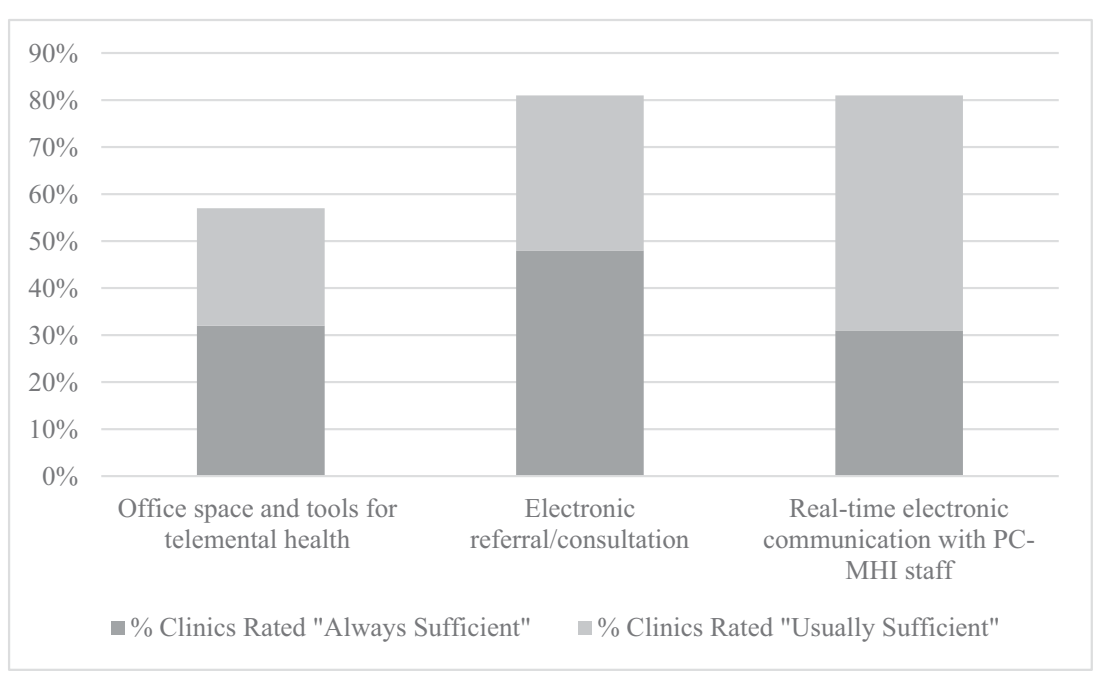

Figure 1 Primary care perceived sufficiency of PC-MHI information communication technology.

(anxiety 71\%, depression 69\%, alcohol use disorder 37\%) (Table 1). Aside from PTSD (68\%), primary care satisfaction was generally lower for non-target mental health conditions, such as substance use disorder (29\%) and serious mental illness (40\%). Half of primary care clinical leaders surveyed reported being "very satisfied" with suicide risk management. A quarter reported being "very satisfied" with care for mental health symptoms related to military sexual trauma and intimate partner violence. Finally, the lowest primary care satisfaction was reported for other behavioral health issues, such as pain (19\%) and sleep problems (36\%).

Availability of care managers for mental health needs was positively associated with primary care clinical leader satisfaction for two PC-MHI target conditions, depression $\left(\chi^{2}=\right.$ $5.66 ; p=.02)$ and anxiety $\left(\chi^{2}=5.38 ; p=.02\right)$ services (Table 1). These associations persisted when controlling for clinic size, distance to VA hospital, and case-mix in both depression $(\mathrm{OR}=8.14 ; 95 \% \mathrm{CI}=1.47-45.02 ; p=.02)$ and anxiety $(\mathrm{OR}=$

Table 1 Primary Care Leader Satisfaction with Mental Health Services Provided. Over the past six months, how would you rate your overall satisfaction with care provided by embedded mental health providers (i.e., availability of care, quality of care, communication related to care, etc.) for your clinic's patients for the following mental health conditions or concerns?

\begin{tabular}{|c|c|c|c|c|c|c|c|c|}
\hline & \multirow[t]{2}{*}{$\begin{array}{l}\text { \% "very satisfied" clinics }(n=59- \\
60)\end{array}$} & \multicolumn{3}{|c|}{$\begin{array}{l}\text { Care manager rated } \\
\text { "available" }(n=55- \\
56)\end{array}$} & \multicolumn{4}{|c|}{$\begin{array}{l}\text { No. of technology components } \\
\text { rated "always sufficient" }(n= \\
53-54)\end{array}$} \\
\hline & & Yes & No & $p$ value & $\mathbf{0}$ & $1-2$ & 3 & $p$ value \\
\hline \multicolumn{9}{|l|}{ Target PC-MHI conditions } \\
\hline Anxiety & $71 \%$ & $45 \%$ & $80 \%$ & $0.02 *$ & $68 \%$ & $84 \%$ & $80 \%$ & 0.44 \\
\hline Depression & $69 \%$ & $40 \%$ & $78 \%$ & $0.02 *$ & $72 \%$ & $74 \%$ & $78 \%$ & 0.95 \\
\hline Alcohol misuse/problem drinking & $37 \%$ & $36 \%$ & $38 \%$ & 0.93 & $32 \%$ & $42 \%$ & $50 \%$ & 0.58 \\
\hline \multicolumn{9}{|l|}{ Non-target mental health conditions } \\
\hline Posttraumatic stress disorder (PTSD) & $68 \%$ & $45 \%$ & $75 \%$ & 0.06 & $76 \%$ & $61 \%$ & $80 \%$ & 0.46 \\
\hline Serious mental illness (schizophrenia, bipolar) & $40 \%$ & $27 \%$ & $42 \%$ & 0.36 & $28 \%$ & $53 \%$ & $60 \%$ & 0.12 \\
\hline Substance misuse/illicit drug problems & $29 \%$ & $36 \%$ & $27 \%$ & 0.52 & $28 \%$ & $21 \%$ & $50 \%$ & 0.26 \\
\hline \multicolumn{9}{|c|}{ Other mental health-linked behavioral health issues } \\
\hline Sleep problems & $36 \%$ & $27 \%$ & $38 \%$ & 0.52 & $40 \%$ & $21 \%$ & $60 \%$ & 0.11 \\
\hline Complex, high medical needs & $29 \%$ & $27 \%$ & $29 \%$ & 0.92 & $20 \%$ & $26 \%$ & $50 \%$ & 0.20 \\
\hline Disruptive behavior & $27 \%$ & $18 \%$ & $29 \%$ & 0.47 & $16 \%$ & $37 \%$ & $40 \%$ & 0.20 \\
\hline $\begin{array}{l}\text { Military sexual trauma, intimate partner } \\
\text { violence }\end{array}$ & $25 \%$ & $18 \%$ & $27 \%$ & 0.56 & $16 \%$ & $32 \%$ & $50 \%$ & 0.12 \\
\hline Medication non-adherence & $22 \%$ & $18 \%$ & $24 \%$ & 0.66 & $12 \%$ & $26 \%$ & $50 \%$ & 0.06 \\
\hline Non-adherence to necessary clinical care & $20 \%$ & $18 \%$ & $22 \%$ & 0.77 & $12 \%$ & $21 \%$ & $40 \%$ & 0.18 \\
\hline Pain & $19 \%$ & $27 \%$ & $18 \%$ & 0.48 & $12 \%$ & $11 \%$ & $50 \%$ & $0.02 *$ \\
\hline Suicide risk assessment and management & $50 \%$ & $36 \%$ & $53 \%$ & 0.31 & $28 \%$ & $74 \%$ & $80 \%$ & $0.002^{\dagger}$ \\
\hline
\end{tabular}

$\underset{\substack{* \\ t p}<.01}{* 0.05}$

Response options dichotomized on a 5-point Likert scale (very satisfied, usually satisfied, neutral, usually dissatisfied, very dissatisfied, not applicable/ did not need services). Response options categorized as number of technology components (telemental health resources, electronic referral/consultation, and instant messaging) rated as "always sufficient". Responses were obtained from 53 to 60 primary care clinical leaders (item response rates were $85-97 \%)$ 
10.07; 95\% CI $=1.50-67.66 ; p=.02)$ outcomes. There was no observed relationship between care managers and primary care satisfaction with other conditions.

\section{PC-MHI Information Communication Technology}

Less than half of primary care clinical leaders reported that resources available for PC-MHI information communication technology as "always sufficient" (Fig. 1). e-consult to PCMHI was highest rated (48\% "always"; $33 \%$ "usually"), followed next by instant messaging with PC-MHI staff (31\% "always"; 50\% "usually"). Office space for tools for telemental health capability was least sufficient of the three technology components assessed (32\% "always"; 25\% "usually"); however, $13 \%$ reported that these resources were not needed. When these three survey items were combined into one scale, $46 \%$ of primary care leaders reported 0 technology components were "always sufficient" (low category), 35\% reported 1-2 components (medium category), and 19\% reported all 3 components (high category).

PC-MHI information communication technology was positively associated with primary care clinical leader satisfaction with suicide risk management $\left(\chi^{2}=12.48 ; p=.002\right)$, even in fully adjusted models $(\mathrm{OR}=3.40 ; 95 \% \mathrm{CI}=1.35-8.57$; $p=.009$ ) (Table 1). We also noted a positive association between PC-MHI information communication technology and primary care satisfaction with pain services $\left(\chi^{2}=8.08\right.$; $p=.02$ ), but this association was not significant after controlling for clinic characteristics. There was no observed relationship between PC-MHI information communication technology and primary care satisfaction with other conditions.

Additionally, we did not find a synergistic effect between availability of care managers and technology on study outcomes.

\section{DISCUSSION}

Primary care clinical leader satisfaction with care from PCMHI providers was high for depression and anxiety, though room for improvement for many other mental and behavioral health issues remained. Depression treatment and anxiety treatment were likely highly rated because they are prevalent mental health conditions for which collaborative care has a well-established evidence base. ${ }^{4}$ PTSD, a condition in which less evidence exists for treatment in primary care, was also highly rated, perhaps because mild PTSD frequently co-occurs with depression, which is managed by PC-MHI. ${ }^{8,27}$ This study and others ${ }^{30}$ have found primary care satisfaction was less than satisfactory for treatment of other conditions (e.g., substance use disorder, pain management), perhaps because accumulating evidence for collaborative care treatment for these conditions has only more recently begun. ${ }^{31,}{ }^{32}$ Despite the existence of brief effective treatments in primary care, ${ }^{33}$ alcohol misuse continues to be the least satisfactory condition targeted by PC-MHI, previously identified as the "greatest current gap" in PC-MHI care by a 2013 VA expert panel. ${ }^{8}$ There is opportunity to use implementation strategies to increase access to effective therapies, such as medical-assisted treatment (MAT) for alcohol use disorder, and to apply the collaborative care model among low-performing conditions, such as designating care managers for chronic pain in Veterans.

Availability of key PC-MHI features, like care managers for mental health needs and information communication technology, holds promise in delivery of high-quality (i.e., providersatisfying) mental health treatment. Primary care clinical leaders representing clinics affected by specialist shortages provided survey input and likely welcome any PC-MHI feature with potential to extend mental health specialist capacity. As expected, care managers were associated with higher primary care satisfaction of mental health services for depression and anxiety. VA studies that informed PC-MHI roll-out (i.e., TIDES, BHL) have long-established evidence for fidelity to models employing care managers for these two common psychiatric illnesses. ${ }^{6,7}$ Evidence-based tools, like care managers for mental health needs, may be further augmented with information communication technology to increase primary care satisfaction for even more conditions, such as timely suicide risk management. Future research will need to explore how to target use of technology in busy clinical settings, especially for conditions requiring urgent attention.

Though not fully envisioned during its inception, primary care clinics are beginning to embrace PC-MHI information communication technology to facilitate access to high-quality mental health care. While primary care clinics continue to ensure the availability of time-tested collaborative care features like care managers for mental health needs, ${ }^{13}$ they have newly begun to embrace technological facilitators of PC-MHI care. While technology sufficiency was not widespread across our primary care study sites, legislative support (VA MISSION Act $)^{22}$ has accelerated its adoption, including more policies and resources that enable patients to receive virtual medical services from home. Existing evidence shows that Veterans are willing to receive technology-based services ${ }^{34}$ and are equally satisfied with virtual (compared to in-person) mental health care. ${ }^{35}$ As capability for telehealth, e-consult, and instant message becomes widespread in primary care clinics, contextual factors become important to end-user perceptions of technological sufficiency. ${ }^{36}$ Our 3 -item scale is among the first to attempt to measure primary care-perceived sufficiency of PC-MHI information communication technology. Ultimately, ensuring sufficient staff interaction with technology (e.g., mental health specialist assigned to telehealth, provider response time to e-consults) remains essential for the successful implementation of these digital innovations.

Our survey reflected the views of nearly all primary care clinical leaders for a VA region serving 1.5 million diverse Veterans in three states; however, there remain several limitations to consider. First, findings reflected only correlations 
between measured variables, in the context of multiple comparisons (i.e., does not imply causation). Second, findings may be subject to unmeasured organizational factors (e.g., staffing and other resources for collaborative care), although we controlled for several observable clinic characteristics. Third, our study did not address clinical decision support, educational tools, or patient-facing technology, which are other potential technologies to enhance PC-MHI care delivery. Finally, our survey data are self-reported and subject to respondent interpretation. Key informants' views may also differ from the views of primary care clinical leaders and providers elsewhere. While our results were obtained from a region where PC-MHI collaborative care services are particularly robust, it may still offer insight into other settings on how to optimally organize health services, such as prioritization of technology for suicide risk management in primary care.

Lessons learned from over a decade of investment in collaborative care models within VA primary care, as highlighted in this current study, may guide other health care systems on how to adjust staffing and technology based on priority conditions. Primary care clinical leader satisfaction with PC-MHI care for depression/anxiety was high, especially among clinics with greater reported availability of care managers for mental health needs. Room for improvement existed among other common conditions (e.g., alcohol misuse, pain). Information communication technology to support PC-MHI care was not consistently implemented across clinics but may facilitate delivery of VA-prioritized suicide risk management. Availability of PC-MHI features (care managers, technology) may, in symbiotic fashion, facilitate practice transformation toward idealized integration of both VA primary care and mental health care. More research is needed to understand the optimal mix of in-person, virtual, and team-based mental health care provided within patient-centered medical homes.

Acknowledgements: The authors would like to thank the Veterans Assessment \& Improvement Laboratory (VAIL) and Mr. Michael G. McGowan (survey administrator), the VISN 22 Primary Care Committee, and former VISN 22 Mental Health Leads (Drs. Peter Hauser and Lucretia Vaughan) for their support of this work.

Corresponding Author: Lucinda B. Leung, MD, PhD, MPH; Division of General Internal Medicine and Health Services Research UCLA David Geffen School of Medicine, Los Angeles, CA, USA (e-mail: lleung@mednet.ucla.edu).

Funding Information This work was undertaken as part of the Veterans Assessment \& Improvement Laboratory (VAIL) for PatientCentered Care (XVA 65-018). Funding is provided by the VA Office of Primary Care to support and evaluate VA's transition to a patientcentered medical home.

\section{Compliance with Ethical Standards:}

The VA Greater Los Angeles Institutional Review Board provided a waiver for this non-research, quality improvement effort.

Conflict of Interest: The authors declare that they do not have a conflict of interest.
Disclaimer: These views represent the opinions of the authors and not necessarily those of the VA or the US government.

\section{REFERENCES}

1. Patient Care Services. Primary Care. https://www.patientcare.va.gov/ primarycare/index.asp. Accessed August 20, 2018.

2. Post EP, Metzger M, Dumas P, Lehmann L. Integrating mental health into primary care within the Veterans Health Administration. Fam Syst Health. 2010;28(2):83-90.

3. Rosland AM, Nelson K, Sun H, et al. The patient-centered medical home in the Veterans Health Administration. Am $J$ Manag Care. 2013;19(7):e263-272.

4. Archer J, Bower P, Gilbody S, et al. Collaborative care for depression and anxiety problems. Cochrane Database Syst Rev. 2012;10:CD006525.

5. Pomerantz AS, Sayers SL. Primary care-mental health integration in healthcare in the Department of Veterans Affairs. Fam Syst Health. 2010;28(2):78-82.

6. Rubenstein LV, Chaney EF, Ober $\mathrm{S}$, et al. Using evidence-based quality improvement methods for translating depression collaborative care research into practice. Fam Syst Health. 2010;28(2):91-113.

7. Oslin DW, Ross J, Sayers S, Murphy J, Kane V, Katz IR. Screening, assessment, and management of depression in VA primary care clinics. The Behavioral Health Laboratory. J Gen Intern Med. 2006;21(1):46-50.

8. Chang ET, Simon A. Report on Integrating Mental Health Into PACT (IMHIP) in the VA. VA Office of Patient Care Services;2013.

9. Daumit GL, Stone EM, Kennedy-Hendricks A, Choksy S, Marsteller JA, McGinty EE. Care Coordination and Population Health Management Strategies and Challenges in a Behavioral Health Home Model. Med Care. 2019;57(1):79-84.

10. Edlund MJ, Young AS, Kung FY, Sherbourne CD, Wells KB. Does satisfaction reflect the technical quality of mental health care? Health Serv Res. 2003;38(2):631-645.

11. Dundon M, Dollar K, Schohn M, Kantinga LJ. Primary Care-Mental Health Integration Co-Located, Collaborative Care: An Operations Manual. Center for Integrated Healthcare;March 2011.

12. Department Of Veterans Affairs. Uniform Mental Health Services in VA Medical Centers and Clinics. VHA Handbook 1160.01. In. Washington, DC2008.

13. Leung LB, Rose D, Stockdale S, et al. Regional Adoption of Primary CareMental Health Integration in Veterans Health Administration PatientCentered Medical Homes. J Healthc Qual. 2019.

14. Levine S, Unutzer J, Yip JY, et al. Physicians' satisfaction with a collaborative disease management program for late-life depression in primary care. Gen Hosp Psychiatry. 2005;27(6):383-391.

15. Ede V, Okafor M, Kinuthia R, et al. An Examination of Perceptions in Integrated Care Practice. Community Ment Health J. 2015;51(8):949-961.

16. Funderburk JS, Sugarman DE, Maisto SA, et al. The description and evaluation of the implementation of an integrated healthcare model. Fam Syst Health. 2010;28(2): 146-160.

17. Clatney L, Macdonald H, Shah SM. Mental health care in the primary care setting: family physicians' perspectives. Can Fam Physician. 2008;54(6):884-889.

18. Benzer JK, Beehler S, Miller C, et al. Grounded theory of barriers and facilitators to mandated implementation of mental health care in the primary care setting. Depress Res Treat. 2012;2012:597157.

19. Benzer JK, Cramer IE, Burgess JF, Jr., Mohr DC, Sullivan JL, Charns MP. How personal and standardized coordination impact implementation of integrated care. BMC Health Serv Res. 2015;15:448.

20. Protti D. Integrated care needs integrated information management and technology. Healthc Q. 2009;13 Spec No:24-29.

21. Steele Gray C, Barnsley J, Gagnon D, et al. Using information communication technology in models of integrated community-based primary health care: learning from the $\mathrm{iCOACH}$ case studies. Implement Sci. 2018;13(1):87.

22. VA Expands Telehealth by Allowing Health Care Providers to Treat Patients Across State Lines [press release]. Washington, DC, May 11, 20182018.

23. Parsons K, Woolley $\mathrm{AB}$. Use of an instant messaging application to facilitate pharmacy students' learning during medical rounds. Am J Health Syst Pharm. 2013;70(19):1654-1655. 
24. Vimalananda VG, Gupte G, Seraj SM, et al. Electronic consultations (econsults) to improve access to specialty care: a systematic review and narrative synthesis. J Telemed Telecare. 2015;21(6):323-330.

25. Fortney JC, Pyne JM, Mouden SB, et al. Practice-based versus telemedicine-based collaborative care for depression in rural federally qualified health centers: a pragmatic randomized comparative effectiveness trial. Am J Psychiatry. 2013;170(4):414-425.

26. Carey TS, Crotty KA, Morrissey JP, et al. In: Future Research Needs for the Integration of Mental Health/Substance Abuse and Primary Care: Identification of Future Research Needs from Evidence Report/Technology Assessment No. 173. Rockville (MD)2010.

27. Rubenstein LV. Integrating Physical and Mental Health Care in the Veterans Health Administration: A Path to the Future. In: Robert Feinstein JC, and Marilyn Feinstein, ed. Integrating Behavioral Health and Primary Care. New York: Oxford University Press; 2017.

28. Raney L, Bergman D, Torous J, Hasselberg M. Digitally Driven Integrated Primary Care and Behavioral Health: How Technology Can Expand Access to Effective Treatment. Curr Psychiatry Rep. 2017;19(11):86.

29. Wagner T, Stefos T, Moran E, et al. Risk Adjustment: Guide to the V21 and Nosos Risk Score Programs. Technical Report 30. Menlo Park, CA: VA Palo Alto;February 2016

30. Kessler R, Miller BF, Kelly M, et al. Mental health, substance abuse, and health behavior services in patient-centered medical homes. $J$ Am Board Fam Med. 2014;27(5):637-644.
31. Kroenke K, Krebs EE, Wu J, Yu Z, Chumbler NR, Bair MJ. Telecare collaborative management of chronic pain in primary care: a randomized clinical trial. JAMA. 2014;312(3):240-248.

32. Watkins KE, Ober AJ, Lamp K, et al. Collaborative Care for Opioid and Alcohol Use Disorders in Primary Care: The SUMMIT Randomized Clinical Trial. JAMA Intern Med. 2017;177(10):1480-1488.

33. Kaner EF, Beyer FR, Muirhead C, et al. Effectiveness of brief alcohol interventions in primary care populations. The Cochrane database of systematic reviews. 2018;2:CD004148.

34. In: Evaluation of the Department of Veterans Affairs Mental Health Services. Washington (DC)2018.

35. Veazie S, Bourne D, Peterson K, Anderson J. Evidence Brief: Video Telehealth for Primary Care and Mental Health Services. 2019.

36. Jackson GL, Krein SL, Alverson DC, et al. Defining core issues in utilizing information technology to improve access: evaluation and research agenda. J Gen Intern Med. 2011;26 Suppl 2:623-627. 